\title{
Patient Participation In Surgical Upper Abdominal Cancer Care: Assessment of The Alignment Between Patients' Preferences And Experiences With The Patient Preferences For Patient Participation (4Ps) Tool
}

Jenny Drott ( $\sim$ Jenny.Drott@liu.se)

Linköping University: Linkopings universitet https://orcid.org/0000-0002-7601-6450

Victoria Fomichov

Unit for Public Health and Statistics, Linköpings University

Maria Nordén

Department of Urology, Linköping University

Anna Lindhoff Larsson

Department of Surgery, Linköping University

\section{Per Sandström}

Department of Surgery, Linköpings University

\section{Bergthor Björnsson}

Department of Surgery, Linköping University

\section{Ann Catrine Eldh}

Department of Health, Medicine and Caring Sciences, Linköping University

\section{Research Article}

Keywords: Cancer care, Involvement, Patient participation, Patient preferences, Person-centred care, surgical care

Posted Date: June 14th, 2021

DOl: https://doi.org/10.21203/rs.3.rs-530855/v1

License: (c) (1) This work is licensed under a Creative Commons Attribution 4.0 International License. Read Full License 


\section{Abstract}

\section{Purpose}

Quality healthcare necessitates opportunities for patient participation. The study aim was to investigate the level of preference-based patient participation in surgical upper abdominal cancer care based on patients' reports of their preferences and experiences of patient participation.

\section{Methods}

A cross-sectional design was used, and patients completed the Patient Preferences for Patient Participation (4P) tool after hospital discharge. Patient ratings of the 12 attributes of the 4Ps in terms of their preferences for and experiences of patient participation were analysed with descriptive and comparative statistical methods.

\section{Results}

The 101 respondents often reported sufficient levels of preference-based patient participation. Having reciprocal communication and being listened to by healthcare staff were commonly deemed crucial for participation. Furthermore, $60 \%$ of the patients suggested that taking part in planning was crucial for their participation, but they experienced this attribute only to some extent.

\section{Conclusion}

To support person-centred participation, further efforts are needed to recognize patients' preferences for involvement; opportunities to share experiences and share in planning healthcare activities are important for patients in surgical upper abdominal cancer care. Probing and recognizing patients' preferences and experiences makes it easier to tailor patient participation to the individual patient, supporting more holistic surgical cancer care.

\section{Introduction}

The World Health Organization [1] highlighted the importance of patient participation, and various countries use the term "patient engagement" in healthcare policies and legislation. While patient participation is advocated throughout healthcare, there are varying conceptualizations of this concept [26], and patient participation is often used interchangeably with patient engagement [7]. For a long time, many studies on patient participation lacked a conceptualization that included the voice of patients; thus, for example, Swedish legislation, which encourages patient participation based on the individual's preferences and condition, defined patient participation mainly as patients receiving information and taking part in decisions regarding their healthcare [8]. However, more recent research suggests a wider understanding of the concept, including a sense of mutual sharing of experiences and engaging in selfcare activities, in addition to engaging in planning and procedures [9-13]. 
Previous studies among patients who had surgery for upper abdominal cancer have suggested the need for further improvements in regard to patient participation [14, 15]. Ibrahim et al. [14] found that even when patients had received information preoperatively, they felt that the provision of the information in a single event could be overwhelming. Currently, limited attention is given to patient participation consistent with the needs and resources of the individual, that is, preference-based patient participation, among patient groups undergoing surgery for upper abdominal cancer. Rather, previous studies have shown that patients favour more information and the visibility of care goals/planning during the surgical cancer care period $[14,15]$. Even though healthcare professionals in surgical cancer care also rate the provision of information as the most important issue for patient participation, they often lack the time to provide information, as the prioritization of other work activities hampers an ideal situation of patient participation [16].

The wider conceptualization of patient participation has received limited attention in the care of people with upper abdominal cancer, potentially jeopardising conditions for preference-based patient participation. Thus, this aim of this study was to investigate preference-based patient participation in surgical upper abdominal cancer care based on patients' reports of their preferences and experiences of patient participation.

\section{Methods}

\section{Design}

This study used a cross-sectional design.

\section{Setting and sample}

To investigate and understand preference-based patient participation in surgical cancer care, patients were recruited from a surgical clinic at a university hospital in southern Sweden. A consecutive selection procedure was used for the inclusion of patients who underwent surgery for upper abdominal cancer. The inclusion criteria were patients over 18 years of age with malignant tumours in the liver, bile ducts, pancreas, or stomach who were treated with elective surgery. The exclusion criteria were patients with cognitive impairment or who did not have mastery of the Swedish language (determined by a research nurse). The flow of patient selection and data collection are illustrated in Fig. 1. No patients were recruited during the summer vacation period.

Informed consent was obtained by a research nurse preoperatively. The written information stated that the study was voluntary and that the patient could withdraw consent and discontinue participation at any time without giving a reason and without this decision influencing the care or treatment given.

Confidentiality was guaranteed throughout the study. The study was performed in accordance with the ethical principles of the Declaration of Helsinki [17], and the study was approved by the Regional Ethics Review Board (No. 2018/413-32).

\section{Data collection and procedure}


Between May 2019 and March 2020, the Patient Preferences for Patient Participation (4Ps) tool was sent to consenting patients two to three weeks after discharge along with a prepaid return envelope to return the completed questionnaire. Of the 132 consenting patients, a total of 101 (77\%) responded with a completed questionnaire.

\section{The 4Ps tool}

The 4Ps tool was developed for the Swedish healthcare context [18]. It was developed based on the results of studies in which patients conceptualized patient participation, in addition to concept analyses in the semantic, lexical, legislative, ethical and theoretical literature [18]. The 4Ps tool has been tested for face validity and psychometric aspects and found to be valid (after adjustments) and appropriate for conceptualizing patient participation [18-20]. It has been further suggested that the tool allows the assessment of patient preferences for engagement [21].

The 4Ps tool comprises two sections, both including the same twelve attributes of patient participation that assess mutual sharing of knowledge (three items), opportunities to understand symptoms and procedures (three items), shared decision-making (three items), and self-care management (three items). All items and their order in the 4Ps tool are presented in Supplementary Table 1. Conceptually, the items relate to the "sharing of" [experience and knowledge] (items 1-6) and "sharing in" [activities] (items 712), reflecting the essence of participation - that is, "to share" [11].

Patients complete the 4Ps tool as follows:

- In Sect. 1, the patient indicates the extent to which each item represents his or her preferences for patient participation using one of four response alternatives: that the attribute is "unimportant", "somewhat important", "very important" or "crucial" [for my experience of participation].

- In Sect. 2, the patient defines the extent to which he or she has experienced patient participation regarding each attribute. Again, there are four response alternatives for each item: "not at all”, "to some extent", "to a large extent", or "entirely".

While the sections can be analysed separately, together they provide a measure of preference-based patient participation [22]. To assess whether the responses in Sect. 1 and 2 indicate "good" conditions for patient participation, the degree to which the patient's responses for each item match or mismatch between the two sections is determined: preference-based patient participation is indicated by a match between the patient's preferences and experiences. The match scores are categorized into three levels of preference-based patient participation, as further outlined in Supplementary Fig. 1:

- Insufficient provision of preference-based patient participation, calling for significant progress (scores of $0-1$ ),

- Fair provision, indicating room and need for improvement (scores of 2-3), and 
- Sufficient provision signifies a complete or near match of patient preferences and experiences (scores 4-5) [22].

\section{Statistical analysis}

The demographic variables are presented as descriptive statistics with counts and proportions. All the patients' preferences and experiences are presented as proportions. The levels of preference-based patient participation were related to each other; therefore, multivariate analysis was performed. The scores defined the three levels of preference-based patient participation, as outlined in Supplementary Fig. 1.

Individual multivariate confidence intervals for proportions with Bonferroni adjustment were calculated for two of the levels, insufficient and sufficient. In this analysis, only the patients who answered all the items in both the preferences and the experiences sections were included.

The two items with the highest frequency and the two items with the lowest frequency of "crucial" patient ratings were identified. The results of the comparison of attributes rated as "crucial" for participation with the patients' responses regarding their experiences of these attributes are described as proportions with confidence intervals with Bonferroni adjustment within each item to cover multiple comparisons.

The 95\% confidence intervals are presented. The diagrams present the items in the same order as they appear in the questionnaire. All analyses were carried out in IBM SPSS Statistics 27 (SPSS Inc., Chicago, IL, USA).

\section{Results}

The results are based on a total of 101 completed questionnaires. The response rate was $76.5 \%$. Table 1 shows the characteristics of the consenting patients. 
Table 1

Patient characteristics.

\begin{tabular}{|c|c|c|}
\hline \multicolumn{2}{|l|}{ Variable } & \multirow{2}{*}{$\begin{array}{l}\text { n (\%) } \\
48(47.5)\end{array}$} \\
\hline Sex & Female & \\
\hline & Male & $53(52.4)$ \\
\hline \multirow[t]{3}{*}{ Age } & -59 & $15(14.9)$ \\
\hline & $60-69$ & $35(34.6)$ \\
\hline & 70- & $51(50.5)$ \\
\hline \multirow[t]{5}{*}{ Education } & Compulsory school through grade 9 & $33(35.1)$ \\
\hline & Two years of high school, trade school & $14(14.9)$ \\
\hline & High school, 3-4 years/University, college & $44(46.8)$ \\
\hline & Other & $3(3.2)$ \\
\hline & Missing & 7 \\
\hline \multirow[t]{4}{*}{ Marital status } & Married/partner & $75(76.5)$ \\
\hline & Single & $22(22.4)$ \\
\hline & Other & $1(1)$ \\
\hline & Missing & 3 \\
\hline \multirow[t]{3}{*}{ Children } & Yes & $84(87.5)$ \\
\hline & No & $12(12.5)$ \\
\hline & Missing & 5 \\
\hline \multirow[t]{4}{*}{ Tumour } & Pancreas & $41(40.6)$ \\
\hline & Liver & $50(49.5)$ \\
\hline & Bile duct & $9(8.9)$ \\
\hline & Stomach & $1(1)$ \\
\hline
\end{tabular}

Regarding patients' preferences for participation, the items "having reciprocal communication" and "being listened to by the healthcare staff" were most often considered crucial for patient participation. In addition, "learning about what is planned for me" was a commonly preferred attribute of participation, more so than "having explanations of my symptoms/issues" and "learning to manage symptoms". The attributes found to be least preferred for participation were "phrasing personal goals", "my experiences being recognized", "managing self-care", and "taking part in planning". All details in terms of the patients' preferences for patient participation are presented in Fig. 2a. 
As described and summarized in Fig. 2b, the patients reported experiencing the highest levels of patient participation (that is, experiencing the attribute entirely or to a large extent) for the following four items: "being listened to", "having reciprocal communication", "having explanations of what is done for me", and "learning of plans". The four items with the lowest levels of patient participation were "taking part in planning", "phrasing personal goals", "learning to manage symptoms" and "managing self-care".

The comparison of the patients' preferences and experiences indicated the levels of preference-based patient participation that had been achieved with the patients. As illustrated in Fig. 3 , the highest match was identified for "being listened to" by healthcare staff: $73 \%$ of the patients had experiences that matched their preferences. On the other hand, for "learning to manage my symptoms", almost one-fifth of the patients had an insufficient match - there was a $19 \%$ mismatch between preferences for and experiences of participation. The two attributes most often considered crucial for participation, "being listened to by the healthcare staff" and "having reciprocal communication", were also the two attributes with the lowest proportions of insufficient levels of preference-based patient participations, but these results were not statistically significant.

The four items with the highest percentages of insufficient preference-based patient participation are presented in Fig. 4. Only patients experiencing an insufficient match between preferences and experiences for the different attributes were included in this analysis (10 to 19 patients out of 101). "Learning to manage symptoms" had the highest percentage (63\%) of insufficient preference-based patient participation (crucial for participation yet experienced only to some extent), while $62 \%$ of the patients rated the item "phrasing personal goals" as very important for my sense of participation but had not experienced any conditions for this attribute at all. Regarding "managing self-care", $58 \%$ stated that this attribute was "crucial" (for the patient to sense that he or she participated), yet the patients experienced this condition only to some extent during the care period. In addition, $60 \%$ of the patients had a high preference for "taking part in planning" (i.e., indicated that the item was crucial for patient participation), but they experienced this attribute only to some extent.

Most patients considered "having reciprocal communication" to be crucial (for their participation), and $94 \%$ of the patients also experienced conditions matching their preferences, experiencing reciprocal communication either to a large extent (fair) or entirely (sufficient). "Being listened to by the healthcare staff" was the item that had the second largest percentage of patients who considered this attribute to be crucial (preference for participation) and also experienced this attribute "to a large extent" (a fair level of preference-based patient participation) or "entirely" (sufficient preference-based participation): $91 \%$ had a sufficient or fair match between their preferences and experiences. Furthermore, the attributes "my experiences being recognized" and "phrasing personal goals" had the lowest matches between preferences and experiences, with only $32 \%$ and $33 \%$ of the patients having sufficient preference-based patient participation for these attributes, respectively (Fig. 5).

\section{Discussion}


The study aim was to investigate preference-based patient participation in surgical upper abdominal cancer care based on patient's reports of their preferences and experiences of patient participation. "Having reciprocal communication" and "being listened to by the healthcare staff" were the attributes identified as most important for this group of patients in terms of their participation. Furthermore, these preferences were also best matched with the patients' experiences. This finding indicates that surgical cancer care may provide preference-based patient participation. However, whether the provision of such participation is unintentional requires further investigation.

The patients in the study reported the lowest preferences and experiences for "phrasing personal goals" and "taking part in planning". These results may be related to their short time in the hospital and may also be associated with the care process, which is often conducted according to standardized programs (fast track) in which goal targets are set for diagnosis or surgical intervention. Goals and fast track planning are usually visible only to staff but may need to be clarified, made visible and communicated with patients to a greater extent. Previous studies have indicated that although phrasing goals corresponds to the concept of patient participation, it is difficult to execute, particularly if patients are suffering from severe or long-term conditions [12].

The results show that "learning to manage symptoms", "taking part in planning" and "phrasing personal goals" are areas that require further attention to meet patients' preferences. "Learning to manage symptoms" was the attribute with the highest proportion of insufficient matches, and "phrasing personal goals" had a relatively high percentage of mismatches in this study. These may be important attributes to improve in clinical practice, especially as previous studies have shown that proper monitoring of the needs of patients with cancer can enhance their ability to cope with the stressful situation and improve their health-related quality of life [23]. Furthermore, there are indications that although professionals aim to provide information relevant to the patient, this effort does not always correspond with the patient's need for information [24]. There are opportunities to better understand patients' preferences for patient participation by means of a more mutual mode in healthcare professionals' communication [25].

The items "learning to manage symptoms" and "phrasing personal goals" were generally considered by the patients to be very important for participation, but the patients did not have experiences that matched their preferences. This result is consistent with a previous study that described that patient involvement in complex cancer surgery as being stimulated by patient-centred care and the provision of support in asking questions and good communication [26].

"Being listened to by the healthcare staff" was sufficiently matched between preferences and experiences for $73 \%$ of the patients, which is a relatively high percentage. However, in person-centred cancer surgical care, all patients should be listened to on their own terms, but $27 \%$ of the patients did not have a match between their preferences for and actual experiences being listened to. Creating a better match between preferences and experiences requires addressing the potential barriers in the healthcare context and those of the individual, including a recognition of the patient's health literacy and prior knowledge and experience [27]. 
"Managing self-care" was rated as crucial or very important for a sense of participation by $80 \%$ of the patients, and $70 \%$ also indicated that they had experienced this attribute entirely or to a large extent. Studies have shown that healthcare professionals' assessment of patients' self-care needs is an unmet care need at each stage of the cancer trajectory to support patients in managing symptoms and self-care $[28,29]$.

The present study has both strengths and limitations. Considering the vulnerability of this group of patients, the $77 \%$ response rate was good, and there were few internal missing values. However, the sample included more patients with pancreatic and liver malignancies and fewer patients with bile duct or gastric cancer, but this distribution was representative of the patients who underwent cancer surgery during the enrolment period. Furthermore, the 4Ps tool is a relatively new tool but has been suggested to be promising for capturing preferences for patient participation [21]. As such, it reflects a broader notion of patient participation and thus corresponds to person-centred patient participation [30].

Although patient participation is considered vital, there are challenges to meeting each patient's personal needs. Participation through involvement in decisions raises ethical issues [31], including the choice to have surgery or not. Due to the severe medical diagnoses of the participants in this study, we provided them with opportunities to conceptualize their preferences and experiences from a wider range of attributes (although still corresponding to the same concept) $[8,18,32]$. Previous studies among patients who had surgery for upper abdominal cancer have suggested further improvements in regard to patient participation [14, 15]; for example, they noted that patients, despite receiving information preoperatively, sensed that the provision of information in a single event could be stressful and overwhelming. Even though healthcare professionals in surgical cancer care rate the provision of information as the most important issue for patient participation [16], they often lack the time to provide information, as the prioritization of other activities hinders an ideal patient participation situation.

\section{Practice Implications}

Although patient participation is considered vital, there are challenges to meeting each patient's personal needs. The findings of our study may provide a basis for further practice implications and interventions and thus sufficient preference-based patient participation in cancer surgical care and in cancer surgical nursing care. Further interventions to support a mutual exchange of patient preferences and experiences are warranted.

\section{Conclusion}

Due to both the matches and mismatches between patients' preferences for and experiences of patient participation in surgical cancer care, there is potential for professionals to recognize both what works and what needs to be improved to adopt a more holistic approach and thus scaffold person-centred care. Having opportunities for reciprocal communication and being listened to by the healthcare staff were rated as critical attributes by patients within the cancer care trajectory. 


\section{Declarations}

Funding: This work was supported by grants from the Medical Research Council of Southeast Sweden (FORSS-862001).

Conflicts of interest/Competing interests: The authors declare that they have no conflicts of interest.

Ethics approval: The study was performed in accordance with the ethical principles of the Declaration of Helsinki, and the study was approved by the Regional Ethics Review Board (No. 2018/413-32).

Consent to participate: Informed consent was obtained.

Consent for publication: Not applicable

Availability of data and material: All data can be found with request of the corresponding author.

Code availability: All analyses were carried out in IBM SPSS Statistics 27 (SPSS Inc., Chicago, IL, USA).

Author's contributions: Jenny Drott: Conceptualization, Methodology, Data collection, Writing- Original Draft, Review/Editing, Project Administration and Supervision; Victoria Fomichov: Methodology, Formal analysis, Software, Data Curation, Visualization; Maria Nordén: Data collection, Writing- Original Draft, Review/Editing

Anna Lindhoff Larsson: Data collection and administration, Writing- Review/Editing; Per Sandström: Data collection, Writing- Review/Editing; Bergthor Björnson: Data collection, Writing- Review/Editing

Ann Catrine Eldh: Conceptualization, Methodology, Writing- Review/Editing

\section{Acknowledgements}

We would like to thank the patients who participated in the study and responded to the questionnaires after their surgical cancer care process.

\section{References}

1. World Health Organization (2013) Exploring patient participation in reducing health-care- related safety risks. Copenhagen, Denmark: WHO Regional Office for Europe

2. Cahill J (1996) Patient participation: a concept analysis. J Adv Nurs 24:561-71.

https://doi.org/10.1046/j.1365-2648.1996.22517.x

3. Longtin Y, Sax H, Leape LL, Sheridan SE, Donaldson L, Pittet D (2010) Patient participation: current knowledge and applicability to patient safety. Mayo Clin Proc 85:53-62 https://doi.org/10.4065/mcp.2009.0248 
4. Nordgren S, Fridlund B (2001) Patients' perceptions of self-determination as expressed in the context of care.J Adv Nurs 35:117-25. https://doi.org/10.1046/j.1365-2648.2001.01828.x

5. Larsson IE, Sahlsten MJ, Segesten K, Plos KA (2011)Patients' perceptions of nurses' behaviour that influence patient participation in nursing care: a critical incident study. Nurs Res Pract 2011:534060. https://doi.org/10.1155/2011/534060

6. Sahlsten MJ, Larsson IE, Sjöström B, Plos KA (2008)An analysis of the concept of patient participation. Nurs Forum 43:2-11. https://doi.org/10.1111/j.1744-6198.2008.00090.x

7. Finset $A$ (2017) Patient participation, engagement and activation: increased emphasis on the role of patients in healthcare. Patient Educ Couns 100:1245-6. https://doi.org/10.1016/j.pec.2017.05.011

8. Eldh AC, Ekman I, Ehnfors M (2010) A comparison of the concept of patient participation and patients' descriptions as related to healthcare definitions. Int J Nurs Terminol Classif 21:21-32. https://doi.org/10.1111/j.1744-618X.2009.01141.x

9. Kvæl LAH, Debesay J, Langaas A, Bye A, Bergland A (2018) A concept analysis of patient participation in intermediate care. Patient Educ Couns 101:1337-50. https://doi.org/10.1016/j.pec.2018.03.005

10. Nilsson M, From I, Lindwall L (2019) The significance of patient participation in nursing care - a concept analysis. Scand J Caring Sci 33:244-51. https://doi.org/10.1111/scs.12609

11. Eldh AC (2019) Facilitating patient participation by embracing patients' preferences-A discussion. J Eval Clin Pract 2019;25:1070-3. https://doi.org/10.1111/jep.13126

12. Luhr K, Holmefur M, Theander K, Eldh AC (2018) Patient participation during and after a selfmanagement programme in primary healthcare - the experience of patients with chronic obstructive pulmonary disease or chronic heart failure. Patient Educ Couns 101:1137-42 https://doi.org/10.1016/j.pec.2017.12.020

13. Årestedt L, Martinsson C, Hjelm C, Uhlin F, Eldh AC (2019)Patient participation in dialysis care-A qualitative study of patients' and health professionals' perspectives. Health Expect 22:1285-93 https://doi.org/10.1111/hex.12966

14. Ibrahim F, Sandström P, Björnsson B, Larsson AL, Drott J (2019) 'I want to know why and need to be involved in my own care...': a qualitative interview study with liver, bile duct or pancreatic cancer patients about their experiences with involvement in care. Support Care Cancer 27:2561-7 https://doi.org/10.1007/s00520-018-4548-8

15. Larnebratt A, Fomichov V, Björnsson B, Sandström P, Lindhoff Larsson A, Drott J (2019)Information is the key to successful participation for patients receiving surgery for upper gastrointestinal cancer. Eur $\mathrm{J}$ Cancer Care (Engl) 28:e12959. https://doi.org/10.1111/ecc.12959 
16. Andersson Å, Wilhelmsson M, Fomichov V, Larsson A, Bjornsson B, Sandström P, Drott J (2020)Patient involvement in surgical care- Health care personnel views and behaviour regarding patient involvement Scand J Caring Sci 35:96-103. https://doi.org/10.1111/scs.12823

17. World Medical Association (2013) World Medical Association Declaration of Helsinki: ethical principles for medical research involving human subjects. JAMA 310:2191-4 https://doi.org/10.1001/jama.2013.281053

18. Eldh AC, Luhr K, Ehnfors M (2015) The development and initial validation of a clinical tool for patients' preferences on patient participation--The 4Ps. Health Expect 18:2522-35

19. Luhr K, Eldh AC, Nilsson U, Holmefur M (2018) Patient preferences for patient participation: psychometric evaluation of The 4Ps tool in patients with chronic heart or lung disorders. Nord $\mathrm{J}$ Nurs Res 38:68-76. https://doi.org/10.1177/2057158517713156

20. Luhr K, Eldh AC, Theander K, Holmefur M (2019) Effects of a self-management programme on patient participation in patients with chronic heart failure or chronic obstructive pulmonary disease: a randomized controlled trial. Eur J Cardiovasc Nurs 18:185-93.

https://doi.org/10.1177/1474515118804126

21. Jerofke-Owen T, Garnier-Villarreal M, Fial A, Tobiano G (2020)Systematic review of psychometric properties of instruments measuring patient preferences for engagement in health care. J Adv Nurs https://doi.org/10.1111/jan.14402

22. Eldh AC, Holmefur M, Luhr K, Wenemark M (2020) Assessing and reporting patient participation by means of patient preferences and experiences. BMC Health Serv. Res. 20:702 https://doi.org/10.1186/s12913-020-05574-y

23. Abu Sharour L, Malak M, Subih M, Bani Salameh A (2020) Quality of life, care needs, and information needs among patients diagnosed with cancer during their treatment phase. Psychol Health Med 25:2528 https://doi.org/10.1080/13548506.2019.1699660

24. Fruijtier AD, Visser LNC, Bouwman FH, Lutz R, Schoonenboom N, Kalisvaart K, et al (2020) What patients want to know, and what we actually tell them: the ABIDE project.Alzheimers Dement (N Y) 6:e12113. https://doi.org/10.1002/trc2.12113

25. D'Agostino TA, Atkinson TM, Latella LE, Rogers M, Morrissey D, DeRosa AP, et al (2017)Promoting patient participation in healthcare interactions through communication skills training: a systematic review. Patient Educ Couns 100:1247-57. https://doi.org/10.1016/j.pec.2017.02.016

26. Thaysen HV, Lomborg K, Seibaek L (2019)Patient involvement in comprehensive, complex cancer surgery: perspectives of patients, relatives and health professionals. Eur J Cancer Care (Engl) 28:e13071 https://doi.org/10.1111/ecc.13071

Page 12/16 

literacy. Nurs Forum 54:315-27. https://doi.org/10.1111/nuf.12331

28. Lee JY, Jang Y, Kim S, Hyung WJ (2020) Uncertainty and unmet care needs before and after surgery in patients with gastric cancer: a survey study. Nurs Health Sci 22:427-35.

https://doi.org/10.1111/nhs.12677

29. Gustavell T, Sundberg K, Langius-Eklöf A (2020) Using an interactive app for symptom reporting and management following pancreatic cancer surgery to facilitate person-centered care: descriptive study. JMIR Mhealth Uhealth 8:e17855.https://doi.org/10.2196/17855

30. Pettersson ME, Öhlén J, Friberg F, Hydén LC, Wallengren C, Sarenmalm EK, et al (2018)Prepared for surgery - communication in nurses' preoperative consultations with patients undergoing surgery for colorectal cancer after a person-centred intervention. J Clin Nurs 27:2904-16 https://doi.org/10.1111/jocn.14312

31. Castro EM, Van Regenmortel T, Vanhaecht K, Sermeus W, Van Hecke A (2016) Patient empowerment, patient participation and patient-centeredness in hospital care: a concept analysis based on a literature review. Patient Educ Couns 99:1923-39. https://doi.org/10.1016/j.pec.2016.07.026

32. Eldh AC, Ekman I, Ehnfors M (2006) Conditions for patient participation and non-participation in health care. Nurs Ethics 13:503-14. https://doi.org/10.1191/0969733006nej898oa

\section{Figures}

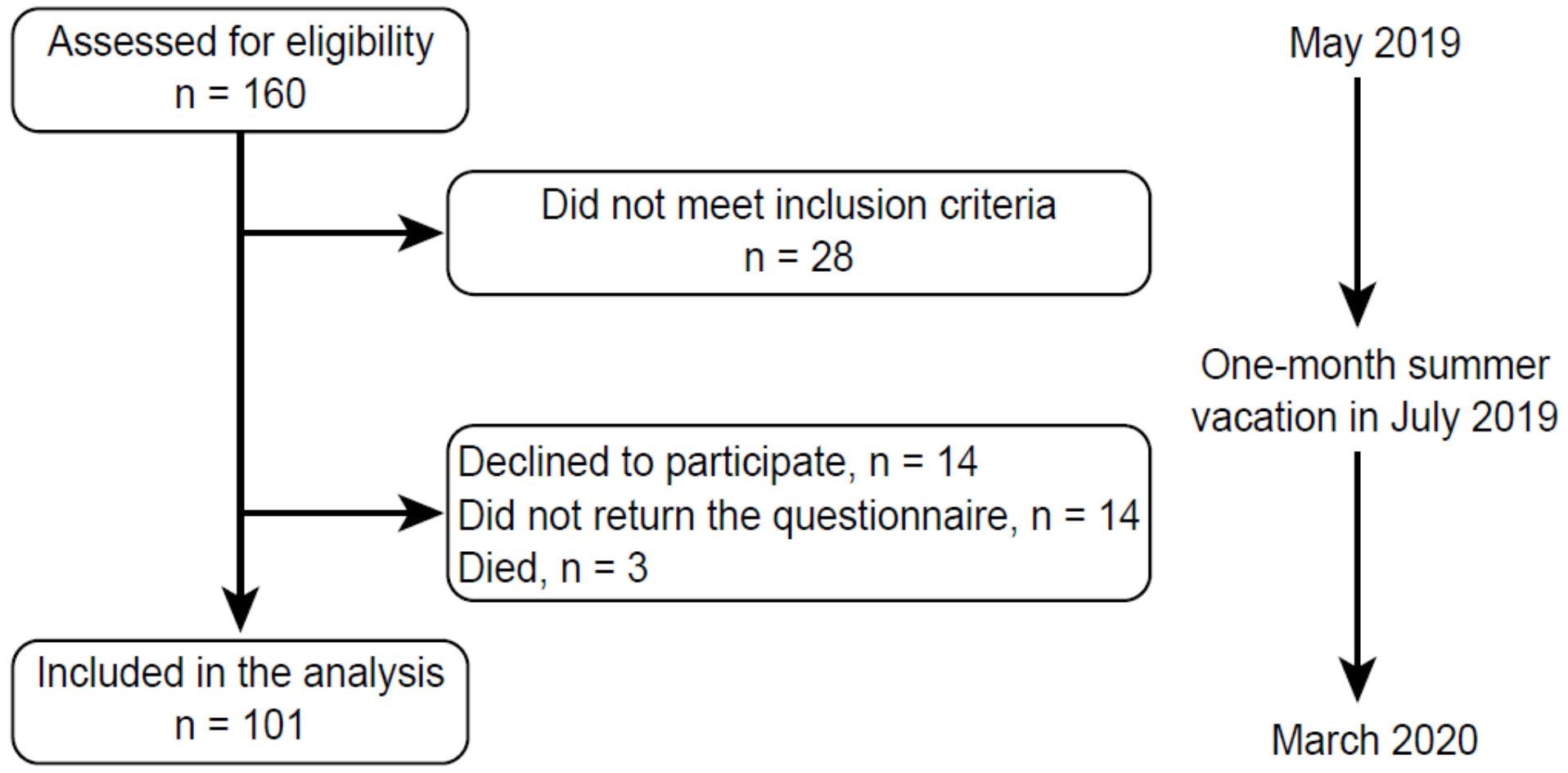


Figure 1

Flowchart of the inclusion process.
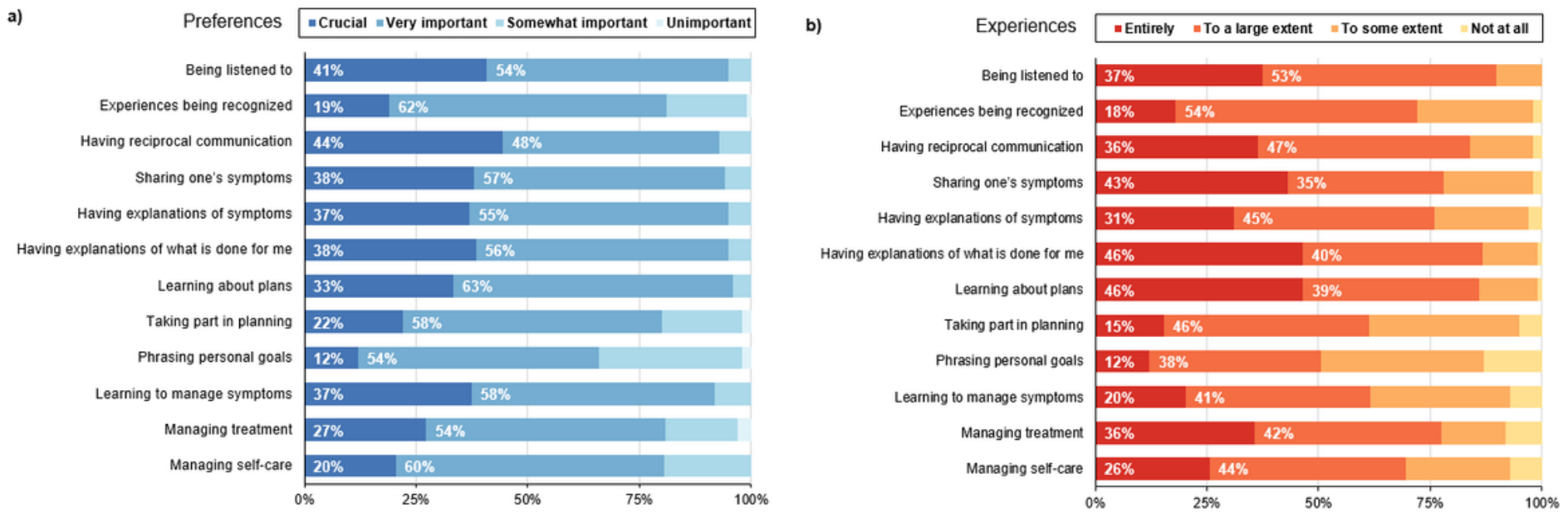

Figure 2

a) Patients' preferences for patient participation for each attribute b) Patients' experiences of patient participation for each attribute (the items are presented in the same order as they are presented in the 4Ps tool).

\section{Insufficient}

Sufficient

Being listened to

Experiences being recognized

Having reciprocal communication

Sharing one's symptoms

Having explanations of symptoms

Having explanations of what is done for me

Learning about plans

Taking part in planning

Phrasing personal goals

Learning to manage symptoms

Managing treatment

Managing self-care

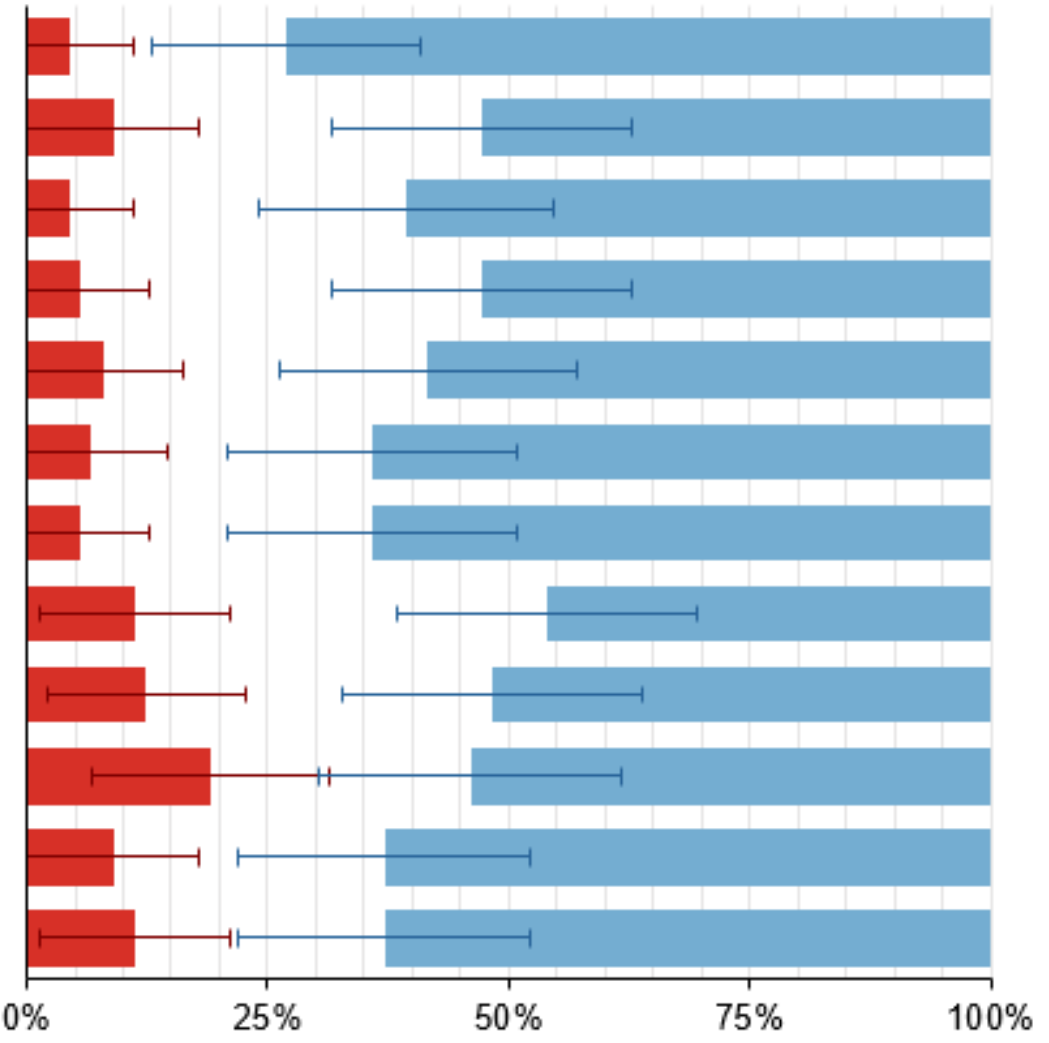

Figure 3 
Insufficient and sufficient levels of preference-based patient participation (individual multivariate $95 \%$ confidence intervals with Bonferroni adjustment, $n=89$ ).

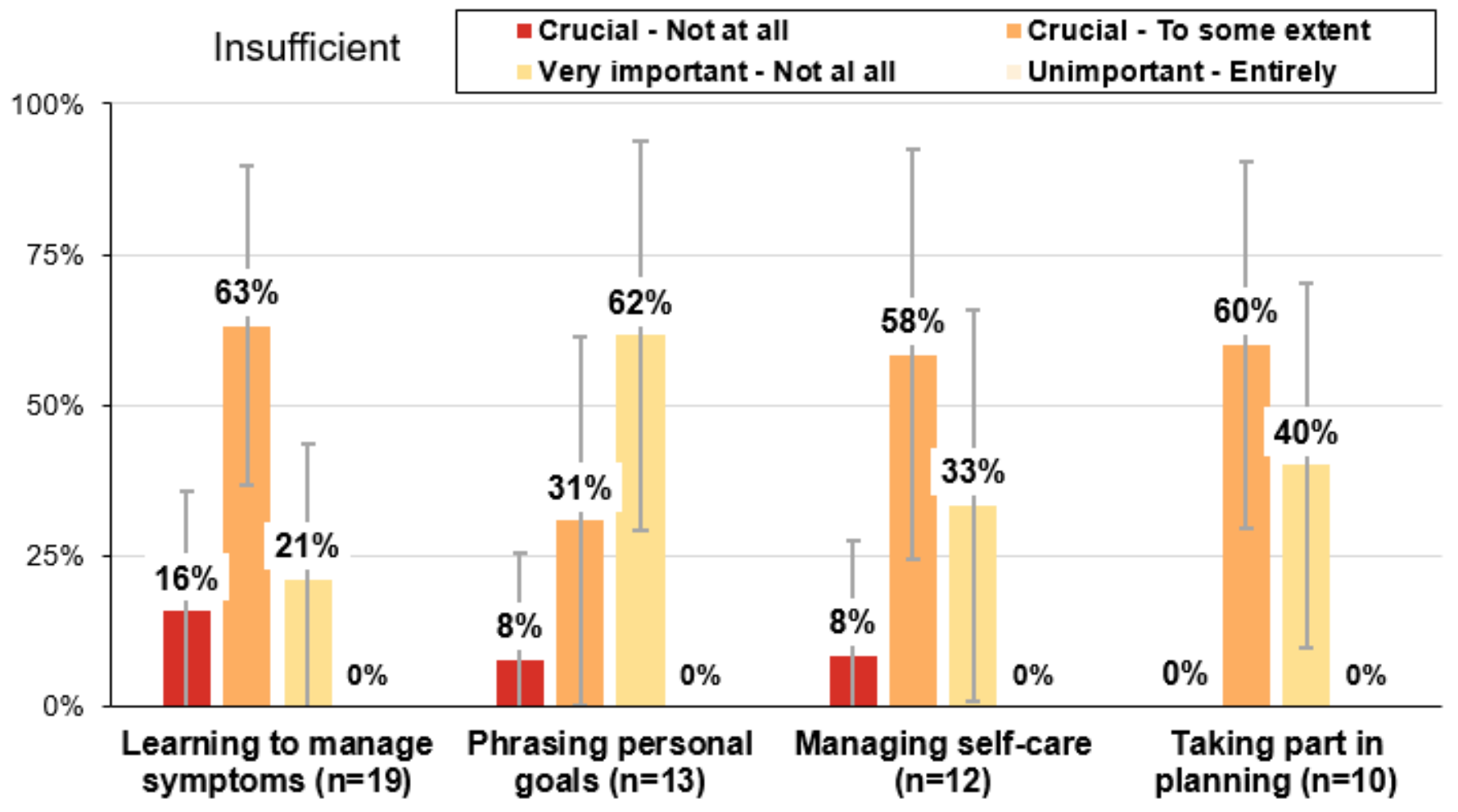

Figure 4

The four attributes of patient participation with the largest percentages of insufficient preference-based patient participation (95\% confidence interval with Bonferroni adjustment for each item).

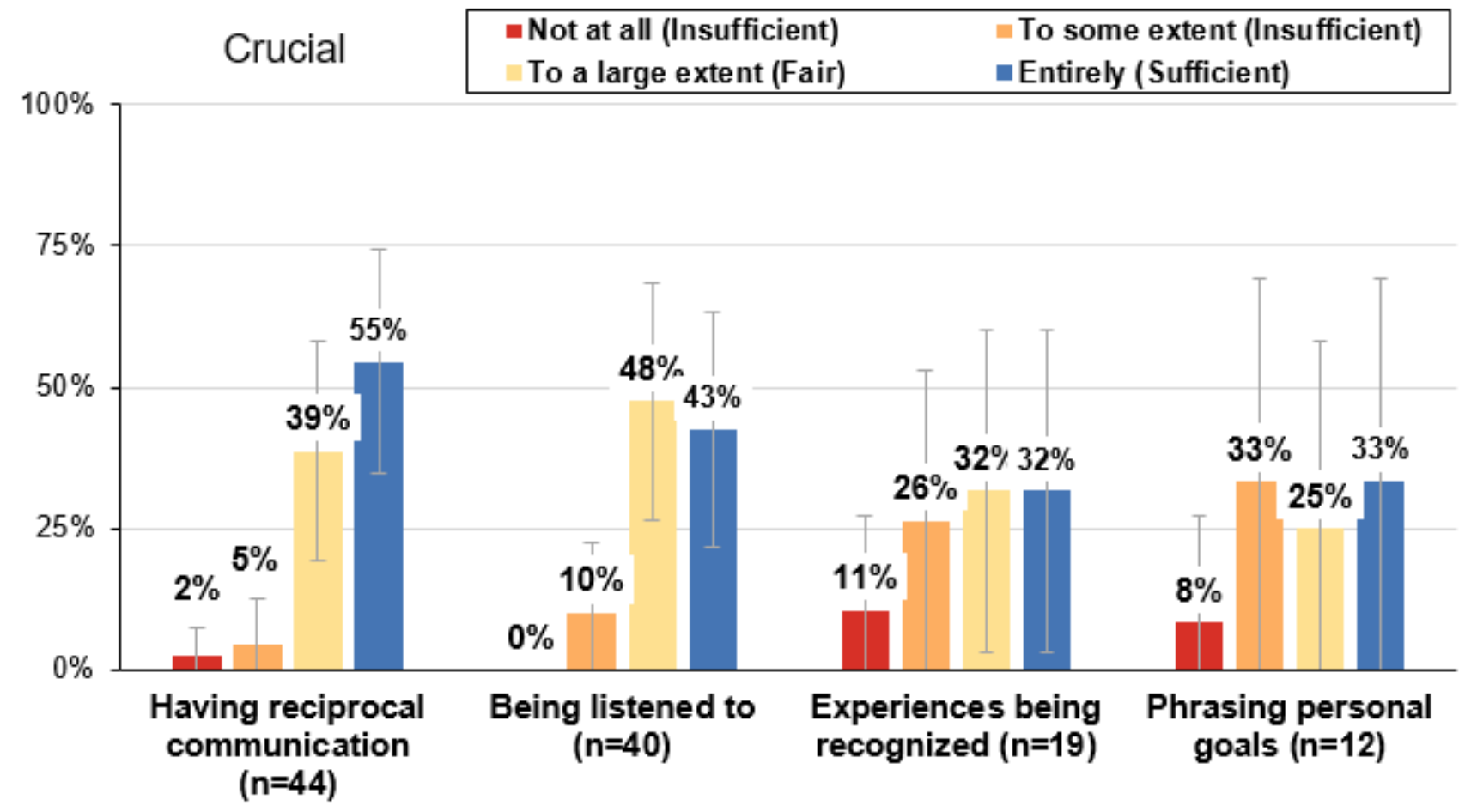




\section{Figure 5}

The two attributes rated as crucial for participation with the highest percentages of sufficient matches and the two attributes rated as crucial for participation with the lowest percentages of sufficient matches (95\% confidence interval with Bonferroni adjustment for each item).

\section{Supplementary Files}

This is a list of supplementary files associated with this preprint. Click to download.

- SupplementaryFigure1.docx

- SupplementaryTable1..docx 